\title{
Infectious Aortitis: Could Early Radiology Improve Patient Outcome?
}

\author{
Anna Døssing ${ }^{1}$, Nick Mattsson ${ }^{2}$ \\ ${ }^{1}$ Department of Rheumatology, Sealand University Hospital, Køge, Denmark \\ ${ }^{2}$ Department of Medicine, Sealand University Hospital, Køge, Denmark
}

Received: 24/09/2018

Accepted: $15 / 10 / 2018$

Published: 08/11/2018

How to cite this article: Døssing A, Mattsson N. Infectious aortitis: could early radiology improve patient outcome? EJCRIM 2018;5: doi:10.12890/2018_000967.

Conflicts of Interests: The Authors declare that there are no competing interests.

This article is licensed under a Commons Attribution Non-Commercial 4.0 License

\section{ABSTRACT}

Aortitis is a rare condition and easily overlooked. It is defined as infectious or non-infectious inflammation of the aortic wall. This report describes two cases of aortitis, demonstrating the diagnostic difficulty and how diagnostic delay could have been reduced if early radiology had been performed. Due to the nature of aortitis, patient outcome can be improved considerably by timely diagnosis and treatment.

\section{LEARNING POINTS}

- Aortitis is rarely suspected due to its vague symptoms, but diagnosis is aided by computed tomography (CT) of the thorax and abdomen.

- A delay in aortitis diagnosis can be life threatening; however, early radiology can ensure timely diagnosis and considerably improve patient outcome.

- Aortitis should always be suspected in cases with immunosuppression and systemic Salmonella infection, and a CT of the thorax and abdomen should be performed.

\section{KEYWORDS}

Aortitis, Salmonella, mycotic aneurysm, infectious arterial aneurysm, aortic ulcer

\section{INTRODUCTION}

Aortitis is inflammation of the aortic wall. It is most commonly caused by rheumatic disease but can also be due to infectious microorganisms ${ }^{[1]}$. Symptoms are closely related to the pathophysiological response. The inflammation can cause fatigue, arthralgia, weight loss and fever, which is often accompanied by leucocytosis and elevated acute phase proteins. The vascular site-specific inflammation leads to pain projected to the back, chest or abdomen, depending on the affected vesse ${ }^{[1,2]}$. As initial symptoms can be vague and are often misinterpreted as non-specific, diagnostics are often delayed and challenging. Computed tomography (CT) is used to exclude mimicking diseases and can also support the diagnosis of infectious aortitis ${ }^{[1]}$. Blood cultures are essential in determining bacterial aetiology ${ }^{[3]}$.

\section{CASES}

Case A was a 69-year-old North European Caucasian male who was admitted with stomach pain, sore muscles, exertional dyspnoea, cough and reduced muscle power. The patient had travelled to Thailand 4 months before hospitalisation and had subsequently experienced 3 months of fluctuating fever and anorexia leading to a $12 \mathrm{~kg}$ weight loss. The patient was receiving active immune-modulating treatment for rheumatoid arthritis with 25 mg methotrexate in tablet form per week and an infusion of infliximab 500 mg every eighth week. The last infusion had been given 4 weeks before hospitalisation. Upon arrival at the hospital, the patient's respiration rate was 28 with an oxygen saturation of $97 \%$ and he had a fever of $38.2^{\circ} \mathrm{C}$ with normal blood pressure and pulse. His general physical examination was normal. Blood 
samples revealed a C-reactive protein (CRP) of $225 \mathrm{mg} / \mathrm{l}$ without leucocytosis. Chest x-ray, electrocardiogram (ECG) and urine examination were normal. Blood cultures showed Salmonella enteritidis on the second day of admission. Antibiotic treatment was initiated in accordance with the antibiotic resistance pattern. However, CRP levels remained above $200 \mathrm{mg} / \mathrm{l}$. Due to the patient's weight loss, a CT of the thorax and abdomen was performed to screen for malignancies 2 days after admission, but the results were not evaluated until day 5 following the request of a physician because of a lack of CRP response. The CT of the thorax and abdomen showed changes diagnostic for infectious aortitis (Fig. 1). The result was confirmed by a positron-emission tomography (PET) CT (Fig. 2). Subsequently surgery was performed for a contained rupture with removal of the infected area and insertion of a venous autograft. Antibiotic therapy consisted of 2 weeks of combination therapy followed by 8 weeks of monotherapy. The patient recovered completely and has now been allowed to continue immune-modulating therapy for rheumatoid arthritis.

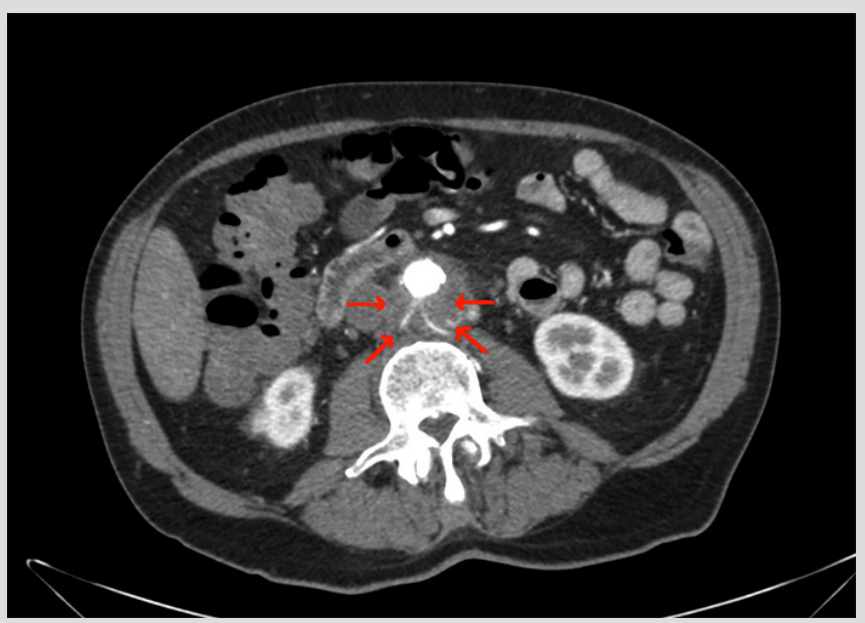

Figure1. Case A. CT of the abdomen with contrast in arterial phase. Cross-section. Posterolateral of the aorta hypodense changes are visible surrounding an $11 \mathrm{~mm}$ aneurysm (red arrows)

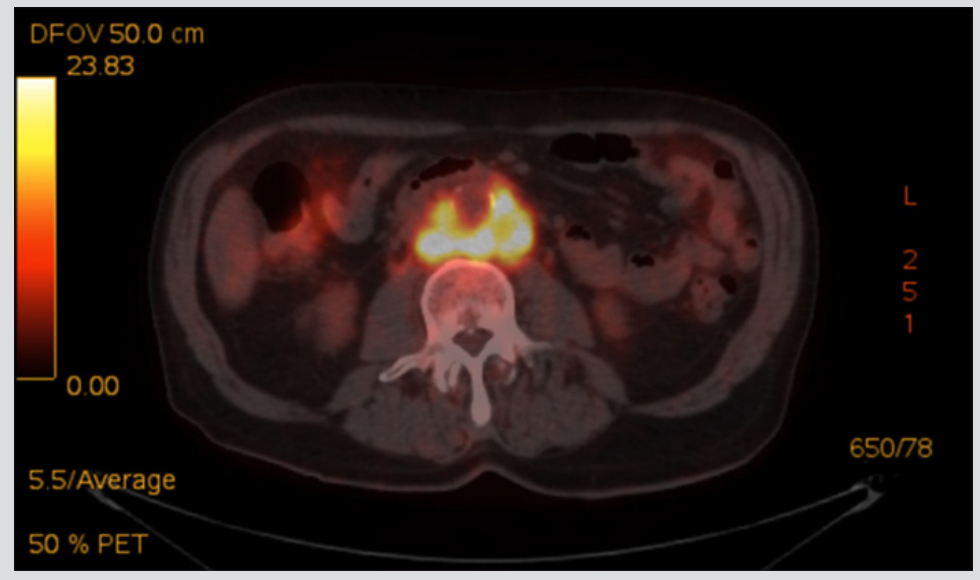

Figure 2. Case A. PET-CT of the abdomen. Cross-section. The picture shows increased metabolic activity in the tissue surrounding the aorta

Case B was an 85-year-old North European Caucasian male with stable chronic lymphatic leukaemia (CLL) requiring no medication, who was admitted with 3 weeks of coughing and 2 days of vomiting, lack of appetite and stomach pain. The patient had returned from a holiday in Bali 3 days before hospitalisation. He had a fever of $38.8^{\circ} \mathrm{C}$, but other vital signs and the physical examination were normal, including ECG. Blood tests showed a CRP of $171 \mathrm{mg} / \mathrm{l}$ and leucocytes of $3.9 \times 10^{\circ} / \mathrm{l}$. Although the chest x-ray was normal, treatment for pneumonia was initiated. The day after admission, blood cultures showed Salmonella enteritidis and so antibiotic therapy was changed accordingly. The patient continued to vomit and experience stomach pain. Four days after admission, the stomach pain had spread to his back and the patient needed morphine for pain relief. CRP remained above $150 \mathrm{mg} / \mathrm{l}$ despite targeted antibiotic therapy. Complicating infectious aortitis was suspected and an acute CT of the abdomen confirmed the diagnosis including retroperitoneal ulcerating lesions (Fig. 3). After acute transfer to the surgery ward, antibiotics were continued for a week to ensure control of the infection and endovascular stenting of the affected aortic area was performed. Post-operative antibiotics were given as monotherapy for 7 weeks and the patient recovered completely. 


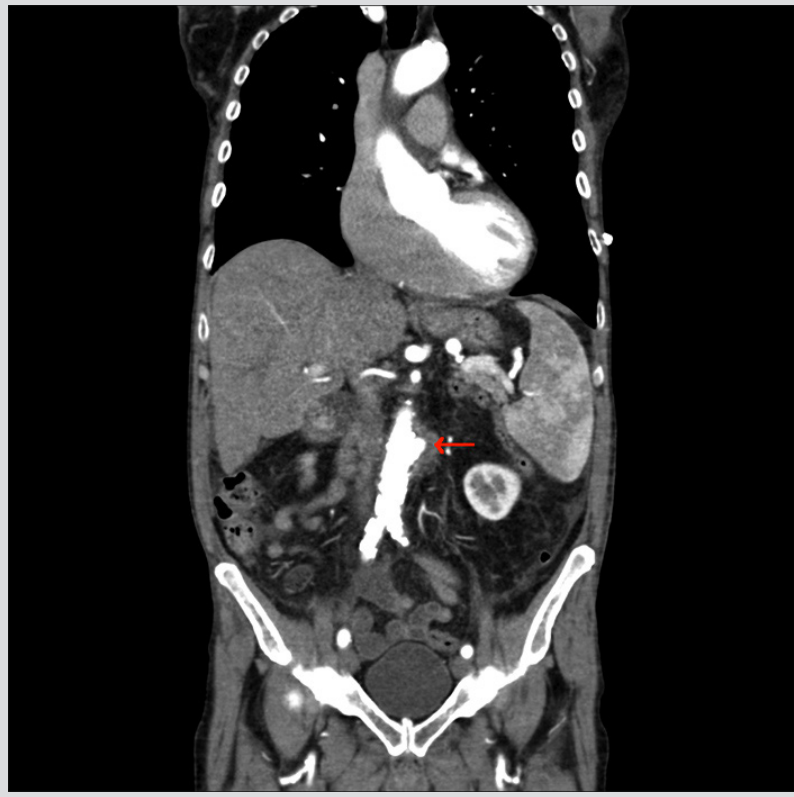

Figure 3. Case B. CT of the thorax and abdomen with contrast in arterial phase. Coronal plane. An infrarenal aortic aneurysm of $3,3 \mathrm{~cm}$ is visible towards the left (red arrow). The wall surrounding the aneurysm is oedematous thickened

\section{DISCUSSION}

Infectious aortitis is considered a rare disease with a low incidence ${ }^{[1,4]}$, but as the condition may be missed, incidence could be higher. Case $A$ presented with non-specific inflammatory symptoms in contrast to case B who presented with gastrointestinal-specific symptoms including pain. Both cases had blood cultures revealing Salmonella species and consistently high CRP despite targeted antibiotic therapy. One third of infectious aortitis is caused by Salmonella species ${ }^{[3]}$, but in $15-50 \%$ of the cases, blood cultures are negative ${ }^{[2]}$.

Risk factors for infectious aortitis include damaged vessels (e.g. atherosclerosis) and a compromised immune system ${ }^{[1,2,4]}$. Risk factors were present in both of our cases. Case A had rheumatoid arthritis and was receiving immune-modulating therapy with methotrexate/infliximab, while case B had CLL. Despite the coexistence of the risk factors of Salmonella in blood cultures, clinical presentation and symptoms, CT evaluation of the chest and abdomen was delayed by 4 and 5 days, respectively, delaying diagnosis and subsequent surgery.

Infectious aortitis is a life-threatening condition and without surgery mortality is $96 \%{ }^{[5]}$. Therefore, correct diagnosis is essential to ensure a combined strategy of antibiotic treatment and surgical intervention. There is no consensus on the duration of antibiotic therapy. Surgical intervention often requires open surgery, but endovascular treatment can be considered as well ${ }^{[1,4]}$.

Gornik et al. suggested in $2008^{[1]}$ that in elderly patients presenting with sepsis or bacteraemia due to Salmonella, CT should be performed immediately to exclude aortitis; we believe that the present cases underline the clinical relevance of this diagnostic tool.

We argue that in cases with coexisting Salmonella infection, immunosuppression and high or resistant biochemical inflammation markers, early radiology, such as abdominal CT, should be considered for timely diagnosis, immediate treatment and subsequently improvement of prognosis.

\section{REFERENCES}

1. Gornik HL, Creager MA. Aortitis. Circulation 2008;117:3039I3051.

2. Stone JR, Bruneval P, Angelini A, Bartoloni G, Basso C, Batoroeva L, et al. Consensus statement on surgical pathology of the aorta from the Society for Cardiovascular Pathology and the Association for European Cardiovascular Pathology: I. Inflammatory diseases. Cardiovasc Pathol 2015;24:2675278.

3. Cabero Moyano J, Andreu Magarolas M, Castañer González E, Gallardo Cistaré X, Belmonte Castan E. Patología aórtica no urgente: diagnóstico clínico-radiológico de la aortitis. RadiologMo 2013;55:4693482.

4. Deipolyi AR, Czaplicki CD, Oklu R. Inflammatory and infectious aortic diseases. Cardiovasc Diagn Ther 2018;8:S618;nds

5. Soravia-Dunand VA, Loo VG, Salit IE. Aortitis due to Salmonella : report of 10 cases and comprehensive review of the literature. Clin Infect Dis $1999 ; 29: 8629868$. 